Check for updates

Cite this: RSC Adv., 2019, 9, 20728

\title{
A new insight into the adsorption-dissolution growth mechanism of zinc oxide hollow hexagonal nanotowers $\dagger$
}

\author{
Rahul Chaudhari, Deepa Landge and Chetan J. Bhongale (D)* \\ The formation mechanism of zinc oxide ( $\mathrm{ZnO}$ ) hollow hexagonal nanotowers ( $\mathrm{h}$-HNTs) synthesized using \\ a simple hydrothermal method was studied. Crystal growth and dissolution mechanism were correlated to \\ the formation of voids and grain boundaries in the nanotowers and are supported by imaging evidence for \\ the first time. Elevated reaction temperature, various ions present in the reaction medium and these voids \\ are responsible for the adsorption-dissolution process and formation of $\mathrm{ZnO}$ h-HNTs.
}

Received 9th May 2019

Accepted 26th June 2019

rsc.li/rsc-advances

stress and the crystallization of $\mathrm{ZnO}$ sheets. It is considered that CTAB can interact with growth units of $\mathrm{ZnO}$ to generate active sites on the surface of ZnO nuclei which grow further. ${ }^{15}$ The cooperative self-assembly between ionic CTAB molecules and inorganic precursor species is developed via electrostatic interaction on which the mineralization of inorganic species strongly depends. ${ }^{14} \mathrm{Xu}$ et al. synthesized $\mathrm{ZnO}$ materials with a range of different morphologies via a simple solvothermal method in which zinc acetylacetonate was used as the zinc source. ${ }^{5 a}$ She et al. proposed that the tubular morphology of $\mathrm{ZnO}$ was formed via the defect selective etching of $\mathrm{ZnO}$ nanowires on the polar surface by protons generated from anodic water splitting: ${ }^{16}$ Several mechanisms like these were proposed investigating the tubular $\mathrm{ZnO}$ morphology, but no detailed study was carried out in this regard.

In this communication, we report the synthesis and formation mechanism of hollow hexagonal nanotowers (h-HNTs) of ZnO. A new concept of 'strong' and 'weak' regions with reference to voids and grain boundaries developed in the nanostructures during solvothermal synthesis of $\mathrm{ZnO}$ is introduced and is supported by imaging and other spectroscopic evidences. Adsorption-dissolution in the context of Kirkendall process is explained at the end. Although the experimental demonstration and theoretical treatment mainly concern binary compounds and planar interfaces or nanoscale spherical interfaces, the fabrication route provided by the Kirkendall effect should be generic, and should also work for high-aspect-ratio hollow cylinders or nanotowers. ${ }^{17}$ XRD results reveal the nanocrystalline wurtzite $\mathrm{ZnO}$ formation through various morphological changes confirmed by scanning electron microscopy (SEM) and matched with that of bulk ZnO standard. Initially, we synthesized ZnO by reacting constituent molecules/precursors at reaction temperature of $200{ }^{\circ} \mathrm{C}$. The reaction mixture was cooled, filtered and dried in the oven at $40{ }^{\circ} \mathrm{C}$ and finally calcined at $550{ }^{\circ} \mathrm{C}$. The SEM images showed the formation of
Department of Applied Chemistry, Defence Institute of Advanced Technology (DU), Girinagar, Khadakwasla, Pune-411 025, Maharashtra, India. E-mail: chetanjb@ diat.ac.in

$\dagger$ Electronic supplementary information (ESI) available: Synthesis of ZnO h-HNTs, characterization, spectroscopic data, etc. See DOI: 10.1039/c9ra03499d 
prominent hollow tubular hexagonal nanostructures tapered at one end (Fig. 1). Fig. 1a and b are that of as-synthesized and calcined $\mathrm{ZnO}$ samples, respectively. The non-uniform distribution of point defects is higher at the center than at the periphery, so the center part etches away faster, which lead to tubular morphology, caused by preferential etching in the (0001) direction. ${ }^{\mathbf{1 6}}$ Nevertheless, it is accepted that the formation of $\mathrm{ZnO}$ nanotubes is kinetically controlled process. A competition between adsorption and dissolution of the precursor molecules, that is crystal growth and dissolution processes determine the final morphology and dimension of the nanostructures. ${ }^{8}$ It is to be noted that the edges of the hexagons were sharp along the tube length. During initial stage, the seeding and growth rate is relatively high because of the high supersaturation degree of growth nutrients. Prolonged solvothermal treatment, at certain equilibrium reaction, the solution composition is no longer thermodynamically favourable for the formation of $\mathrm{Zn}(\mathrm{OH})_{2}$ that subsequently dehydrate into $\mathrm{ZnO} .{ }^{18}$ At this stage (prolonged solvothermal treatment), the rate of $\mathrm{ZnO}$ dissolution is faster as compared to the rate of formation of $\mathrm{Zn}(\mathrm{OH})_{2}$ making it a competing process. ${ }^{10}$ Polar surfaces gets dissolved preferentially since this decreases the system energy during the subsequent aging process, leading to the formation of $\mathrm{ZnO}$ nanotubes. ${ }^{19}\left(\mathrm{Zn}(\mathrm{acac})_{2} \cdot \mathrm{H}_{2} \mathrm{O}\right)$ and CTAB with weight ratio $1: 10$ yielded very fine-textured hollow tubes with a tapered end (towers), and basal hexagons and edges were sharp along the tube length. The preferential etching along $c$ axis and the surfactant (CTAB) protection to the lateral surfaces are considered responsible for the formation of $\mathrm{ZnO}$ hollow nanotowers. ${ }^{7}$ The average thickness of the hexagonal walls of these nanotowers was approximately $70 \mathrm{~nm}$. It is important to note that similar thickness was observed for the wall differentiating 'strong' and 'weak' regions of the solid nanotowers and is discussed in the following section. With this morphology, we assumed the evolution of the nanotowers may be from hexagonal discs formed during the initial stages of solvothermal reaction (see Fig. $3 \mathrm{c}$ for schematic). Wang et al. ${ }^{9}$ proposed that the tower-like structure was produced by the layer-layer stacking of the $\mathrm{ZnO}$ nanosheets. So to verify this assumption and study the growth mechanism and formation of hollow hexagonal nanotowers (h-HNTs), we carried out the control reaction at $80{ }^{\circ} \mathrm{C}$ for $5 \mathrm{~h}$. We collected the small portions $(\sim 20 \mathrm{~mL})$ of the reaction samples at specific time interval (viz., $30 \mathrm{~min}, 1 \mathrm{~h}$ and 5 h) and allowed the reaction to continue for each time. The
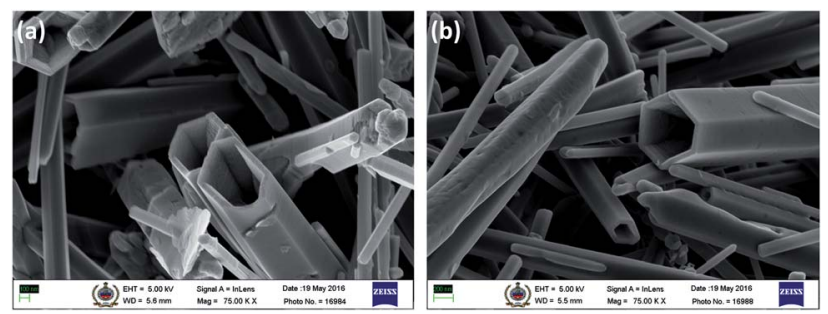

Fig. 1 Scanning electron microscopy (SEM) images at same magnification $(\times 75 \mathrm{k})$ of (a) as-synthesized $\mathrm{ZnO}$ sample synthesized at solvothermal reaction temperature of $200{ }^{\circ} \mathrm{C}$, scale bar $=100 \mathrm{~nm}$ and (b) corresponding sample calcined at $550^{\circ} \mathrm{C}$ for $5 \mathrm{~h}$, scale bar $=200 \mathrm{~nm}$. samples were taken within a very short span of time, centrifuged, filtered and gave washings with warm distilled water (50 ${ }^{\circ} \mathrm{C}$ ) and powders obtained finally dried in oven at $60{ }^{\circ} \mathrm{C}$. The sample thus prepared after $5 \mathrm{~h}$ time interval was termed assynthesized sample, some of which was calcined at $550{ }^{\circ} \mathrm{C}$ for $5 \mathrm{~h}$. Further characterization was done on these samples. SEM images of samples of $\mathrm{ZnO}$ are shown in Fig. 2a-h. These samples showed evolution of nanotower morphology through assembly of nanoparticles.

$\mathrm{ZnO}$ precipitation by hydrolysis of zinc acetylacetonate in an aqueous medium is a rapid chemical process that results in bursting of nuclei followed by $\mathrm{ZnO}$ nanocrystallites formation. Recently, Petrovic et al. ${ }^{20}$ described the growth of stable $\mathrm{ZnO}$ hexagons grown hierarchically, with the aid of CTAB. According to them, with the addition of $\mathrm{NH}_{4} \mathrm{OH}$ to the precipitation system a $\mathrm{Zn}\left(\mathrm{NH}_{3}\right)_{4}{ }^{2+}$ complex is formed, which accelerates the dissolution process of the solid phase and at high autoclaving temperature $\left(220^{\circ} \mathrm{C}\right)$, the decomposition of $\mathrm{Zn}\left(\mathrm{NH}_{3}\right)_{4}{ }^{2+}$ complex takes place forming $\mathrm{Zn}(\mathrm{OH})_{2}$ by a competitive reaction. $\mathrm{Zn}(\mathrm{OH})_{2}$ further transforms to $\mathrm{ZnO}$ nanocrystallites. However, no clear evidence was given for the formation of these lamellar substructures in hexagonal pyramids, except correlating these lamellae with the change in relative intensities of XRD peaks at (100), (002) and (101). We also did observe increase in the
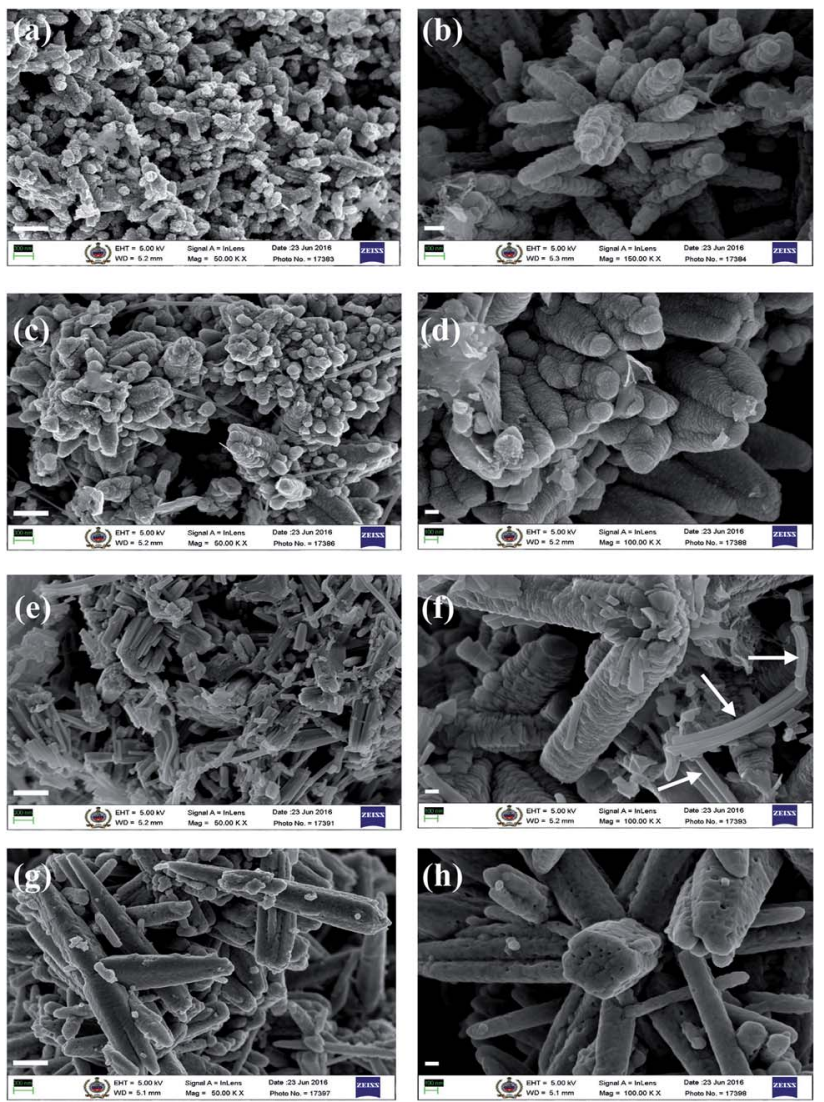

Fig. 2 Scanning electron microscopy images at different magnifications, for samples obtained from the hydrothermal control reaction $\left(80^{\circ} \mathrm{C}\right)$, taken at time interval of ( $\mathrm{a}$ and b) $30 \mathrm{~min}$ (c and d) $1 \mathrm{~h}$, (e and f) $5 \mathrm{~h}$ as-synthesized, and ( $\mathrm{g}$ and $\mathrm{h}$ ) $5 \mathrm{~h}$ as-synthesized sample calcined at $550{ }^{\circ} \mathrm{C}$. Scale bar: $(\mathrm{a}-\mathrm{d})=500 \mathrm{~nm},(\mathrm{e}-\mathrm{h})=100 \mathrm{~nm}$. 
intensities for these diffraction peaks in the XRD pattern, which will be described in the subsequent sections, but control experiment and FE-SEM images of the samples thereof rule out the formation of these hexagonal lamellae.

Initially, after $30 \mathrm{~min}$ and $1 \mathrm{~h}$ of reaction, the $\mathrm{ZnO}$ samples obtained showed hierarchical morphology formed through nanoparticles assembly (Fig. 2a-d). In the growth process, crystals tend to minimize the total surface energy by growing along the [0001] direction. After $5 \mathrm{~h}$ of reaction, mixed morphology (Fig. 2e and f) was visible in the form of hierarchical nanotowers formed by nanoparticles assembly, as well some sheet-like structures (shown by white arrows). Interestingly, the samples calcined at temperature of $550{ }^{\circ} \mathrm{C}$ showed formation of hierarchical hexagonal nanotowers which were not hollow (Fig. $2 \mathrm{~g}$ and $\mathrm{h}$ ). Nanoparticles get coalesced giving smoothening effect to the hierarchical nanotowers obtained earlier. The calcined $\mathrm{ZnO}$ samples were free from sheet-like structures. It was contrary to our assumption of hierarchical assembly of hexagonal discs leading to h-HNTs formation. Thus, nanotowers formed reveal hexagonal shape without lamellar hexagonal stackings. Distinguishing illustration of $\mathrm{ZnO}$ h-HNTs formation mechanism is given in the schematic of Fig. 3c.

Searson et $a .^{21}$ found that several anions in the solution could adsorb on the surface of $\mathrm{ZnO}$ nuclei and had great effects on the coarsening rate and also temperature dependence of the bulk solubility of ZnO. In solution phase synthesis of nanostructures, processes such as coarsening and aggregation can compete with nucleation and growth. The acetate and hydroxyl ions generated during reaction system may also have
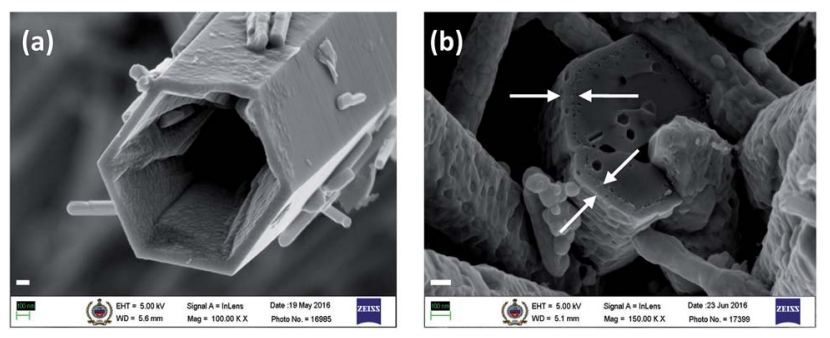

(c)

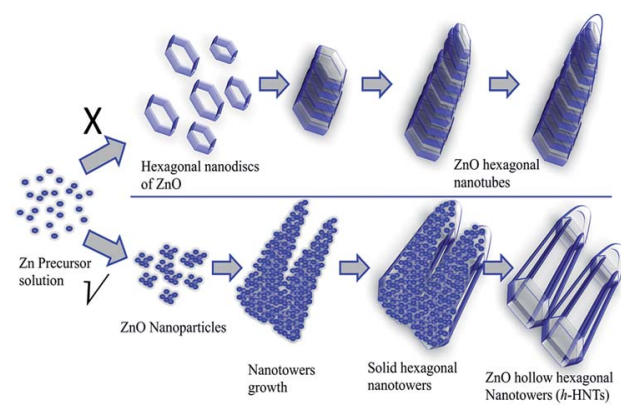

Fig. 3 Scanning electron microscopy images of calcined $\left(550{ }^{\circ} \mathrm{C}\right)$ samples obtained, for comparison, from the solvothermal reactions carried out at (a) $200^{\circ} \mathrm{C}$ and (b) control reaction, $80^{\circ} \mathrm{C}$. Areas denoted by arrows in (b) indicate the boundary differentiating 'strong' and 'weak' regions. (c) Schematic showing the mechanism of formation of $\mathrm{ZnO} \mathrm{h}-\mathrm{HNTS}$ through adsorption and dissolution process. Scale bar = $100 \mathrm{~nm}$. coarsening effect at higher temperature $\left(200{ }^{\circ} \mathrm{C}\right)$ but it might have been minimized at reaction temperature of $80{ }^{\circ} \mathrm{C}$. This could be the reason that we obtained the tapered nanotowers with no etching of center part, at $80{ }^{\circ} \mathrm{C}$ reaction temperature. Formation of $\mathrm{ZnO}$ was observed during initial period itself (30 min) as confirmed by XRD measurements. This shows reaction temperature, consequently vapour pressure, apart from various ions present in solution, plays an important role in the crystal growth and dissolution process in the formation of $\mathrm{ZnO}$ nanotubes. Here we demonstrate the formation of 'weak' and 'strong' regions and subsequent dissolution of 'weak' region generated by formation of voids and grain boundaries as follows.

Porous (voids) nature of the solid $\mathrm{ZnO}$ nanotowers, unlike hexagonal nanotubes, indicates defects and can be partially attributed to the Kirkendall type diffusion. This is evident in SEM magnified image of Fig. 3b (areas denoted by arrows on the base of hexagonal nanotower) which clearly shows the development of thin boundary between periphery of the hexagonal wall and the center core, for the $\mathrm{ZnO}$ synthesized at $80{ }^{\circ} \mathrm{C}$ reaction temperature for $5 \mathrm{~h}$ duration and subsequent calcination. It proves the necessity of elevated reaction temperatures, subsequently increased vapour pressure for the formation of nanotubes. This is probably the first example shown with evidence the crystal growth and dissolution is due to 'weak' and 'strong' regions developed during ZnO nanotowers/nanotubes formation. 'Weak' regions originated because of voids formation, collapses paving way for hollow/tubular morphology. Fig. 3a is the magnified SEM image of the ZnO h-HNTs formed by solvothermal reaction carried out at $200{ }^{\circ} \mathrm{C}$. The tapered hexagonal nanotube structure was retained in the sample calcined at $550{ }^{\circ} \mathrm{C}$ also.

Formation of hollow nanostructures is possible with conversion chemistry with three major classes of conversion chemical reactions: the Kirkendall effect, galvanic exchange, and anion exchange, each of which can result in void formation in the materials. ${ }^{22}$ Several studies on formation of various $\mathrm{ZnO}$ morphologies like hollow nanoparticles, ${ }^{23}$ nanospheres and nanotubes, ${ }^{24}$ nanocages,${ }^{25}$ dandelions, ${ }^{26}$ etc. are attributed to Kirkendall effect. ${ }^{17,27}$ Porous nature of the hierarchical ZnO nanostructures (Fig. 2g, h and 3b) indicate defects (unlike hexagonal nanotubes in Fig. 1) and can be attributed due to the injection rates of vacancies and to some extent Kirkendall type diffusion. The porous nature of the solid nanotowers could also be clearly observed in transmission electron microscopy (TEM) images as shown in ESI Fig. S2(b and c). $\uparrow$ TEM of h-HNTs show transparent tubes image. As mentioned in the preceding section, the solid HNTs show boundary layer at periphery of hexagon and extending vertically along the tower length with number of voids in the core (Fig. 3b). These defects (voids) might aid dissolution of the core leading to hollow tubes/towers formation. Elevated reaction temperature $\left(200{ }^{\circ} \mathrm{C}\right)$, is also required for adsorption-dissolution that takes place during solvothermal synthesis of ZnO. Energy dispersive X-ray (EDX) spectra and $\mathrm{ZnO}$ composition of these samples are shown in ESI Fig. S2 and Table $1, \uparrow$ respectively. EDX measurements shed light on the composition of nanostructures with respect to zinc 
and oxygen. During solvothermal reaction as it proceeds the atomic zinc percentage is decreased, whereas atomic oxygen percentage is increased. Increase in oxygen atomic percentage is likely due to various complexes formed on the nanostructures surfaces by reacting species like zinc acetate, ammonia, CTAB, adsorbed water molecules, etc. $\mathrm{Zn}$ : O stoichiometric atomic ratio of calcined sample of hydrothermal reaction product (80 ${ }^{\circ} \mathrm{C}$ ) solid nanotowers, show $1: 1 \mathrm{Zn}$ : O composition, as evident from SEM-EDX measurements.

Formation and evolution of $\mathrm{ZnO}$ nanotowers was also studied by XRD and phase composition and phase purity at all stages were identified by XRD patterns as depicted in Fig. 4. It shows the formation of $\mathrm{ZnO}$ taking place during initial period itself (30 min) and until $5 \mathrm{~h}$ of duration, well-evolved prominent ZnO peaks appeared with increased intensity. The strong intensities of $\mathrm{ZnO}$ peaks indicate good crystallinity. These samples also showed some peaks in the $2 \theta$ region of 10 to $30^{\circ}$ confirming the presence of $\mathrm{Zn}(\mathrm{OH})_{2}$ and some unknown minor impurities (probably present in the reaction or of CTAB). The XRD peaks $(2 \theta=21,27,33)$ due to $\mathrm{Zn}(\mathrm{OH})_{2}$ diminishes as the reaction proceeds. In the sample $(5 \mathrm{~h})$ calcined at $550{ }^{\circ} \mathrm{C}$, these peaks were disappeared, showing formation of clean $\mathrm{ZnO}$ matching the XRD peaks that of bulk $\mathrm{ZnO}$ as standard. This supports the imaging observation we had for the sheet-like structures in the $5 \mathrm{~h}$ as-synthesized sample and disappearance of the same in the calcined one (Fig. 2); indicating the origin of $10-30^{\circ} 2 \theta$ region peaks may be due to these sheet-like unknown structures and $\mathrm{Zn}(\mathrm{OH})_{2}$. The remarkable increase in the XRD peak intensities for the sample calcined at $550{ }^{\circ} \mathrm{C}$ is attributed to the well-evolved crystalline hexagonal tapered nanotowers. No additional peaks were noticed, validating the pure form of the ZnO. Spectroscopic characterizations like UVPL, FTIR, Raman were carried out for ZnO nanostructures formed at various stages and standard $\mathrm{ZnO}$ and are described in ESI in details (Fig. S3†).

We have fabricated hollow hexagonal nanotowers of $\mathrm{ZnO}$ by simple solvothermal synthesis carried out at elevated

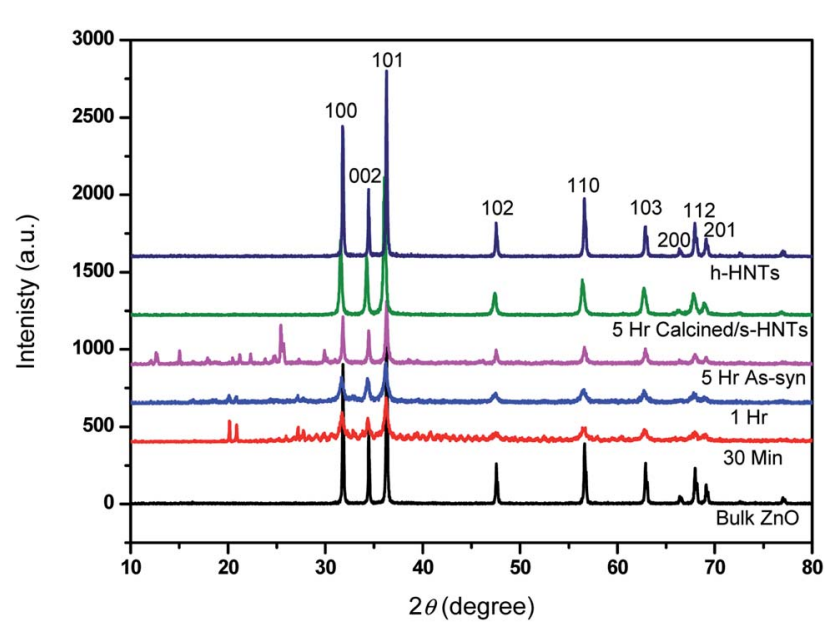

Fig. 4 Powder $\mathrm{X}$-ray diffraction spectra of $\mathrm{ZnO}$ samples collected at various time intervals from control reaction carried out at $80^{\circ} \mathrm{C}$, as indicated. XRD pattern of bulk $\mathrm{ZnO}$ standard is also shown for comparison. temperature of $200{ }^{\circ} \mathrm{C}$. Control reaction at lower temperature shows hierarchical morphology formed by nanoparticles aggregation and upon calcinations, nanoparticles get coalesced giving rise to hexagonal towers which were not hollow. Evolution and growth of $\mathrm{ZnO}$ nanostructures is monitored and explained with a suitable mechanism. Hexagonal nanotubes formed without lamellar hexagonal stackings. It is observed that elevated reaction temperature $\left(200{ }^{\circ} \mathrm{C}\right)$, various ions present in the solution along with nanoscale Kirkendall effect are responsible for adsorption-dissolution process and formation of hollow hexagonal nanotowers of $\mathrm{ZnO}$ during solvothermal synthesis. To the best of our knowledge, mechanism of ZnO h-HNTs or nanotubes evolution with imaging and spectroscopic evidence is reported for the first time by control reaction.

\section{Conflicts of interest}

Authors declare no conflicts of interest.

\section{Acknowledgements}

This work was funded by SERB-DST, Government of India under the research grant SB/FT/CS-056/2013.

\section{References}

1 (a) U. Özgür, Y. I. Alivov, C. Liu, A. Teke, M. A. Reshchikov, S. Doğan, V. Avrutin, S. J. Cho and H. Morkoç, J. Appl. Phys., 2005, 98, 041301; (b) D. C. Look, Mater. Sci. Eng., B, 2001, 80, 383.

2 M. Taghavi, V. Mattoli, B. Mazzolai, C. Filippeschi and L. Beccai, Nanoscale, 2013, 5, 3505.

3 X. Y. Kong, Y. Ding, R. Yang and Z. L. Wang, Science, 2004, 303, 1348.

4 J. Ding, X. Fang, R. Yang, B. Kan, X. Li and N. Yuan, Nanoscale Res. Lett., 2014, 9, 1.

5 (a) L. Xu, Y.-L. Hu, C. Pelligra, C.-H. Chen, L. Jin, H. Huang, S. Sithambaram, M. Aindow, R. Joesten and S. L. Suib, Chem. Mater., 2009, 21, 2875; (b) C. Pacholski, A. Kornowski and H. Weller, Angew. Chem., Int. Ed., 2002, 41, 1188.

6 Q. Zhou, W. Chen, L. Xu and S. Peng, Sensors, 2013, 13, 6171.

7 H. Wang, M. Li, L. Jia, L. Li, G. Wang, Y. Zhang and G. Li, Nanoscale Res. Lett., 2010, 5, 1102.

8 Y. Tong, Y. Liu, C. Shao, Y. Liu, C. Xu, J. Zhang, Y. Lu, D. Shen and X. Fan, J. Phys. Chem. B, 2006, 110, 14714.

9 Z. Wang, X.-F. Qian, J. Yin and Z.-K. Zhu, Langmuir, 2004, 20, 3441.

10 H. Jianga, J. Hub, F. Gua and C. Li, J. Alloys Compd., 2009, 478, 550.

11 K. Yu, Z. Jin, X. Liu, J. Zhao and J. Feng, Appl. Surf. Sci., 2007, 253, 4072.

12 S. Musić and A. Šarić, Ceram. Int., 2012, 38, 6047.

13 Y. Xi, J. Song, S. Xu, R. Yang, Z. Gao, C. Hu and Z. L. Wang, J. Mater. Chem., 2009, 19, 9260.

14 L. Shen, N. Bao, K. Yanagisawa, K. Domen, C. A. Grimes and A. Gupta, J. Phys. Chem. C, 2007, 111, 7280. 
15 H. Zhang, D. Yang, Y. Ji, X. Ma, J. Xu and D. Que, J. Phys. Chem. B, 2004, 108, 3955.

16 G. She, X. Zhang, W. Shi, X. Fan and J. C. Chang, Electrochem. Commun., 2007, 9, 2784.

17 H. J. Fan, M. Knez, R. Scholz, K. Nielsch, E. Pippel, D. Hesse, M. Zacharias and U. Goesele, Nat. Mater., 2006, 627.

18 M. N. R. Ashfold, R. P. Doherty, N. G. Ndifor-Angwafor, D. J. Riley and Y. Sun, Thin Solid Films, 2007, 515, 8679.

19 (a) Q. Li, V. Kumar, Y. Li, H. Zhang, T. J. Marks and R. P. H. Chang, Chem. Mater., 2005, 17, 1001; (b) S. Xu and Z. L. Wang, Nano Res., 2011, 4, 1013.
20 Z. Petrovic, M. Ristic and S. Music, Ceram. Int., 2014, 40, 10953.

21 Z. Hu, G. Oskam, R. L. Penn, N. Pesika and P. C. Searson, J. Phys. Chem. B, 2003, 107, 3124.

22 B. D. Anderson and J. B. Tracy, Nanoscale, 2014, 6, 12195.

23 H. J. Fan, U. Gosele and M. Zacharias, Small, 2007, 3, 1660.

24 A. A. El Mel, R. Nakamura and C. Bittencourt, Beilstein J. Nanotechnol., 2015, 6, 1348.

25 H. Fan, J. Zheng, J. Hu, Y. Su, N. Zhao, J. Xu, F. Liu and F. Pan, Part. Part. Syst. Charact., 2015, 32, 771.

26 B. Liu and H. C. Zeng, J. Am. Chem. Soc., 2004, 126, 16744. 27 P. Afanasiev, J. Phys. Chem. C, 2012, 116, 2371. 